\title{
Optimal design of adaptive power scheduling using modified ant colony optimization algorithm
}

\author{
Vijo M. Joy, S. Krishnakumar \\ School of Technology and Applied Sciences, M G University Research Centre Edappally, India
}

\begin{tabular}{l}
\hline \hline Article Info \\
\hline Article history: \\
Received May 1, 2019 \\
Revised Oct 3, 2019 \\
Accepted Oct 15, 2019 \\
\hline
\end{tabular}

\section{Keywords:}

Ant colony optimization Artificial neural networks Back propagation Load scheduling Power management

\begin{abstract}
For generating and distributing an economic load scheduling approach, artificial neural network (ANN) has been introduced, because power generation and power consumption are economically non-identical. An efficient load scheduling method is suggested in this paper. Normally the power generation system fails due to its instability at peak load time. Traditionally, load shedding process is used in which low priority loads are disconnected from sources. The proposed method handles this problem by scheduling the load based on the power requirements. In many countries the power systems are facing limitations of energy. An efficient optimization algorithm is used to periodically schedule the load demand and the generation. Ant colony optimization (ACO) based ANN is used for this optimal load scheduling process. The present work analyse the technical economical and time-dependent limitations. Also the works meets the demanded load with minimum cost of energy. Inorder to train ANN back propagation (BP) technics is used. A hybrid training process is described in this work. Global optimization algorithms are used to provide back propagation with good initial connection weights.
\end{abstract}

Corresponding Author:

Vijo M. Joy,

Department of Electronics, School of Technology and Applied Sciences,

M G University Research Centre,

Edappally, Cochi- 24, Kerala, India.

Email: vijomjoy@gmail.com

\section{INTRODUCTION}

The hydropower sources are inadequate to meet the required power demand; hence the ideal scheduling of different power sources is important [1]. Thermal sources are very expensive; on the other side, solar energy is easily available. But the amount of power generated from solar power sources is insufficient to meet the load demand. The economical scheduling problems are the 'unit commitment' and 'online dispatch'. In unit commitment, fixed amount of power is supplied through the network for scheduling at minimum cost over a required period of time. Online dispatching means distribution of the power in parallel with the system [2]. To meet the required power, all the available power sources are considered. When considering all these aspects, an optimum load scheduling method is required for energy saving and meeting the power demand with minimum cost. Among the various sources, thermal power is very expensive. Therefore in a practical scheduling approach, a low priority is given to the thermal energy source. When a low cost power source is available, the maximum power is sourced from it and remaining power is contributed from other sources [3].

The purpose of the use like domestic, industrial, agriculture, etc., peak time and non-peak time, emergency conditions, type of use like private or public, holidays or any other important days, and more demanded or less demand area are the factors affecting the use of load. So in this work it is focused to realize 
an optimization method that helps us to consider all the above parameters and generate an optimized path to make a good decision based on the priority. Among these parameters some conditions have high priority and some have low priority. So the ultimate aim is to generate an optimization rule by considering all the parameters and to generate an optimized result. That result helps us to make a time bounded result and to take a decision for scheduling the power source. The most important term in the power system is stability [4].

The instability of a system makes serious threats to the system security. The instability leads to total blackout of the system [1]. The primary aim of most of the studies in this area is mainly focusing to forecast the power based on cost and time. In the existing approaches, instability of the power generation is one of the major drawbacks. With the present method, one can overcome this drawback by scheduling the load based on the requirement. In this method, scheduling is applied at the both sides, i.e., generation - distribution side and consumer side. That is the optimal scheduling of power depends on the load. The total load of the system is the sum of the maximum load required by the entire regions and power lossess. There is no need to make any change in the output of the power plants. In case any region requires additional power to meet its load requirement, the proposed system checks the regions with their maximum load. This comparison helps the system to find the units with minimum load requirement and schedule this load to the required region. That means no interruption is made on the power source [5].

In ANN, the inputs are the maximum load to each region and the additional load required to any region. At the same time, power generated and power losses are the outputs of the system. The scheduling is proposed to be carried out based on Back Propagation Neural Network (BPNN) algorithm [6]. ANN is a popular and fast growing technology and it is used in a wide range of applications not limited to pattern classification, pattern recognition, optimization products and automatic circuits. The ANN is useful to get an optimal solution in various scheduling applications [7]. This work primarily focuses on training ANN for different load demands. Once the training has been completed, the network acquires the ability to generate load scheduling pattern for any load demand.

The power demand for each region and the additional power required to them due to increase in the load and radiation power loss act as the inputs for the neural networks. The total power required for entire section is the output. Complex and nonlinear relations are used by the BPNN for solving and analyzing the problems. Based on the way they learn, there are many types and architecture of neural network. BPNN is a classical multilayer feed forward network trained according to back propagation algorithm [8]. The preference of optimal combination of connection weights are the basis of this work. Sometimes BPNN fails to find the global optimum. Therefore global optimization algorithm ant colony optimization (ACO) is used to predict the better path. To handle the qualitative and quantitative knowledge, BPNN uses parallel distributed processing approach. It has fault tolerance and adaptability, strong robustness, and can fully approximate any complex nonlinear relationship. Because of this dominance, BPNN is more suitable for processing the electric energy signals which is noisy, unstable and nonlinear. For the scheduling process, feed forward neural network trained by back propagation (BP) algorithm is used. This is a supervised learning method; means the neural network create a model from the examples of information with predicted output value. To reduce the error between the reference value and the system output, mean square error is used as the cost function which is given in equation 1. The ACO is used to minimize the cost.

$$
J_{w}=\frac{1}{N} \sum_{k=1}^{N}\left(y_{k}-z_{k}\right)^{2}
$$

Where $y_{k}$ the output of the model and $z_{k}$ is is the reference output.

With the help of the proposed ACO and ANN, the system minimizes the cost and better utilization of all the available power sources (depends on the demand and availability). Implementation of this algorithm focuses to minimize the error. This work also aims to minimise the power generation cost and disturbance. To control the operation time and energy consumption an efficient ACO based algorithm is proposed with low computational complexity. This algorithm is inspired by the organized natural movement of real ants and has been successfully applied to different large optimization problems.

The optimal algorithm for load shedding is proposed by Hooshmand, et.al. [1] In their method, a fast algorithm for load shedding is introduced. They reduced the manual effort by separating the least possible loads from the neural network. A Hydro-thermal scheduling based on ANN was proposed by Suman, et al. [9]. In this scheduling method, the total load is shared between hydro and thermal power stations according to the cost of power generation. An algorithm for energy management system based on multi-layer ant colony optimization is presented to find energy scheduling in Microgrid by Marzband et al [10]. They introduced a calculation strategy for energy utilization system. For the applications in real time energy management, system a regularised particle swarm optimization algorithm is introduced by Hossain et al [11]. 


\section{METHODOLOGY}

\subsection{Neural network}

The ANN is a class of mathematical algorithm designed to solve a specific problem [12]. This is a family of statistical learning models inspired by biological neural networks that are the central nervous systems of animals, in particular the brain. They are used to estimate or approximate functions that can depend on a large number of inputs and are generally unknown. The ANNs are generally presented as systems of interconnected "neurons" which send messages to each other. The connections have numeric weights that can be tuned based on experience, making neural nets, adaptive to inputs, and capability of learning. It is considered as a nonlinear statistical data modelling tool. Neural networks consist of input layer, hidden layer and output layers. It consists of a number of processing nodes which are connected in a network [13]. This node is an imitation of real neuron. Interconnected artificial neurons are used to make the structure of ANN, which are trained to recreate the biological neuron properties. The model of a neuron is shown in Figure 1. The input $\left[x_{1} x_{2, \ldots . .} x_{n}\right]$ their corresponding weights $\left[w_{1}, w_{2,}, \ldots w_{n}\right]$ bias ' $\mathrm{b}$ ' and activation function ' $\mathrm{f}$ ' applied to the weighted sum of the inputs.

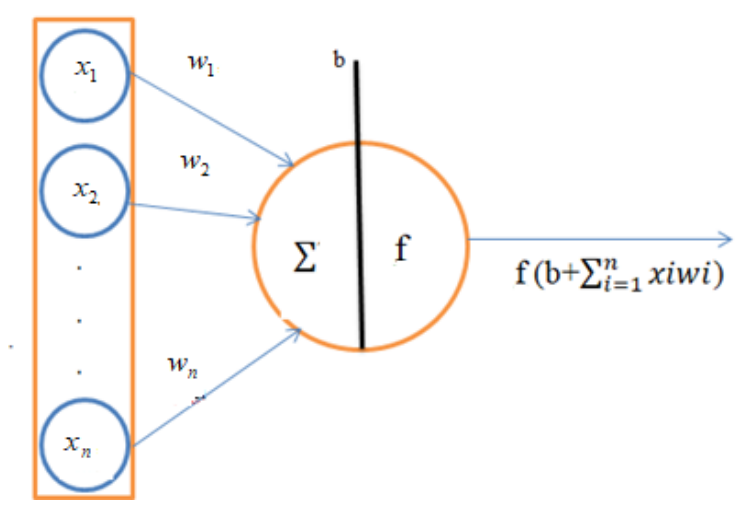

Figure 1. Model of a neuron

\subsection{BPNN}

The BPNN is a local optimization algorithm for training network and uses Levenberg - Marquardt technique [14]. This algorithm takes more memory but its execution time is less. When the generalization stops improving, this algorithm is automatically stopped. The network error is back propagated after comparing with the target value and the actual output of ANN. In Figure 2, $\left[x_{1}, x_{2}, \ldots . x_{n}\right]$ and $\left[y_{1}, y_{2}, \ldots y_{n}\right]$ are the input independent variables and their corresponding output in the BPNN respectively. In addition $\left[a_{1}, a_{2}, \ldots . a_{n}\right]$ are the actual values for the corresponding training data, i.e., the expected output value from the model. The weight corresponding to each node in the hidden layer is $\mathrm{w}_{\mathrm{ij}}$ and the weight corresponding to each node in the output layer is $w_{j k}$. The number of input nodes is ' $n$ '. The number of hidden layer nodes is ' 1 ', the number of output nodes is ' $\mathrm{m}$ ' and the threshold of each node is ' $d$ '. MATLAB is used for training the network.

The output of the hidden layer is

$$
o_{j}=f\left(\sum_{i=1}^{n} w_{i j} x_{i}-d_{j}\right) ; \mathrm{j}=1,2,3 \ldots l
$$

The output of the output layer is

$$
y_{k}=f\left(\sum_{i=1}^{l} o_{j} w_{j k}-d_{k}\right): \mathrm{k}=1,2,3 \ldots \mathrm{m}
$$




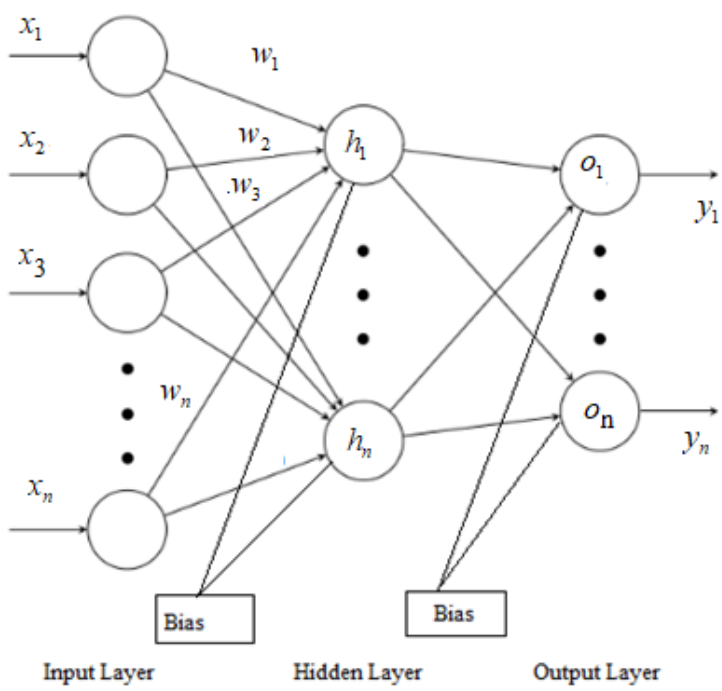

Figure 2. ANN structure

The mean square error $\left(E_{k}\right)$ is calculated using the predicted and the expected output value and is shown in (4).

$$
E_{k}=\frac{1}{2} \sum_{k}\left(a_{k}-y_{k}\right)^{2}
$$

\subsection{ANT colony optimisation}

The ACO is a conventional swarm intelligent technique for interpret combinatorial optimization problems [15]. The main inspiration behind ACO algorithm is the concept of 'stigmergy' in nature. Stigmergy is a method of self-organization and it refers to the modification of environment by biological organisms to communicate with each other. In fact an indirect communication takes place when an individual configure the environment and at a later time others responds to that modified environment [16].

In an ant colony, all the ants are regularly search for the food around the nest in a random manner. 'Pheromone' is used by ants for the local communication. These hormonal chemicals are generated and emitted by an organism to give information to the other members of their species. Ant marks their travelling path with pheromones when they reach at a food source [17]. The quality and quantity of the food source is highly proportional to the amount of emitted pheromones. Ant deposits strong and concentrated pheromone if they found a better and more source of food. Other ants recognize the existence of the pheromone and follow it to reach at the food source $[18,19]$. Ants carry the collected food to their nest and mark their own path to the next. This is how the ants find the shortest path between nest and a food source. Vaporization takes place when ants deposit pheromones. The pheromone updating period is inversely proportional to the length of the path between nest and source of food. It shows that the shortest path become more concentrated as more ants get attracted [20]. Demonstration of this phenomenon updation is illustrated in Figure 3. A finite set of available solutions are used to create artificial ants [21]. Each ant takes values from this finite set. An ant can select the entire unvisited node available from the current position in its each iteration. An ant ' $k$ ' at node ' $r$ ' will choose the destination node 's' at a later stage with a probability,

$$
P_{k(r, s)}=\frac{\tau_{r, s}^{\alpha} \eta_{r, s}^{\beta}}{\sum \tau_{r, s}^{\alpha} \eta_{r, s}^{\beta}}
$$

Where, $\tau_{r, s}$ shows the amount of pheromone of r, s edge, $\alpha$ - defined the impact of pheromone,

$\eta_{r, s}$ indicates the desirability of r,s edge, and $\beta$-defines the impact of the desirability. 


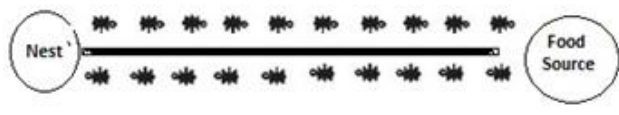

(a)

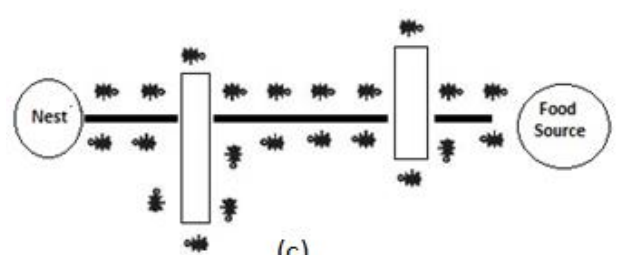

(c)

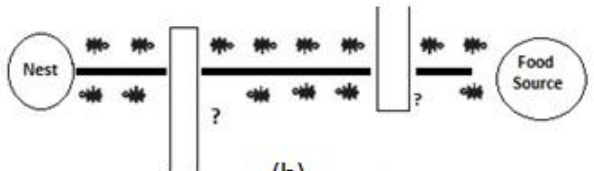

(b)

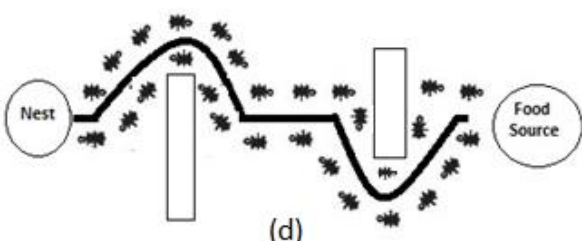

(d)

Figure 3. Modifying behaviour of a real ant colony; (a) search of food; (b) ants follow a path between nest and food source (c) with equal probability, check whether to shortest or longest path;

(d) the shortest path is choosed by majority of ants

The desirability of an edge is defined by a weighting function. This function is mostly heuristic and it assigns a value which shows how good an edge is [22]. If the distance should be minimised, $\eta_{\mathrm{r}, \mathrm{s}}$ can be defined as,

$$
\eta_{r, s}=\frac{1}{d_{r, s}}
$$

Where $d_{r, s}$ is the length of the edge $\mathrm{r}, \mathrm{s}$

The pheromone phase is used for the communication between the artificial ants and it helps to make decisions [23, 24]. Pheromone evaporation and deposit are the major components in this phase. The rate of pheromone on $\mathrm{r}$, s edge is:

$$
\tau_{r, s}=(1-\rho) \tau_{r, s}+\Delta \tau_{r, s}
$$

Where $\rho$ defines the rate of pheromone evaporation and $\Delta \tau_{r, s}$ is the total amount of pheromone deposited on the $r$, s edge which is defined as,

$$
\begin{array}{cl}
\Delta \tau_{r, s}=\frac{1}{L_{k}} & ; \mathrm{k}^{\text {th }} \text { ant travels on edge } \mathrm{r}, \mathrm{s} \\
0 & ; \text { otherwise }
\end{array}
$$

Where $\mathrm{L}_{\mathrm{k}}$ shows the length (cost value) of the path that $\mathrm{k}^{\text {th }}$ ant travels.

In this algorithm, virtual pheromones generated by the artificial ants are used for updating the path. The density and the amount of pheromone are the major factors that affect their path updating [25, 26]. It depends on the comparison of the quality of the pheromone with the former artificial ant of the same iteration. Algorithm 1 explains the training process for ACO-BPNN hybrid network.

Algorithm 1:

a. Initialise the process by setting the parameters like pheromone value, heuristic information and stopping function.

b. Construct the solution for each ant and then evaluate the constructed solutions. Repeat the process until exit criteria met. If the criterion is not met then update the pheromone value using the modified ACO (MACO).

c. On the occasion that exit criterion is satisfied; update the initial weights of BP.

d. Train the BP network and obtain MSE. Again the process checks for the exit criterion.

e. If the condition fails repeat step 4. Otherwise save the results for MSE and Regression. 


\section{RESULTS AND ANALYSIS}

Efficiency for handling the parameters which have random nature is one of the main features of this system. The pheromones act as a memory element. Pheromones get updated after each iteration. This helps to meet the fast growing demand of energy, in addition with the increased generation, improved efficiency, stability and energy conservation. In this paper, a self-determining management of energy at demand-side is presented .This scheduling system encourage the users for reducing their electricity consumption without compromising with service quality and customer satisfaction. Table 1 shows the ACO parameters used for training. Best cost vs number of iterations is shown in Figure 4. When the iteration started the system tries to minimize the cost by updating the pheromones. The system optimized to the minimum cost within the iteration limit.

Table 1. ACO parameters

\begin{tabular}{ll}
\hline \multicolumn{1}{c}{ ACO parameters } \\
\hline Maximum Number of Iterations & 20 \\
Number of Ants (Population Size) & 10 \\
Initial Pheromone & 1 \\
Pheromone Exponential Weight & 1 \\
Heuristic Exponential Weight & 1 \\
Evaporation Rate & 0.05 \\
\hline
\end{tabular}

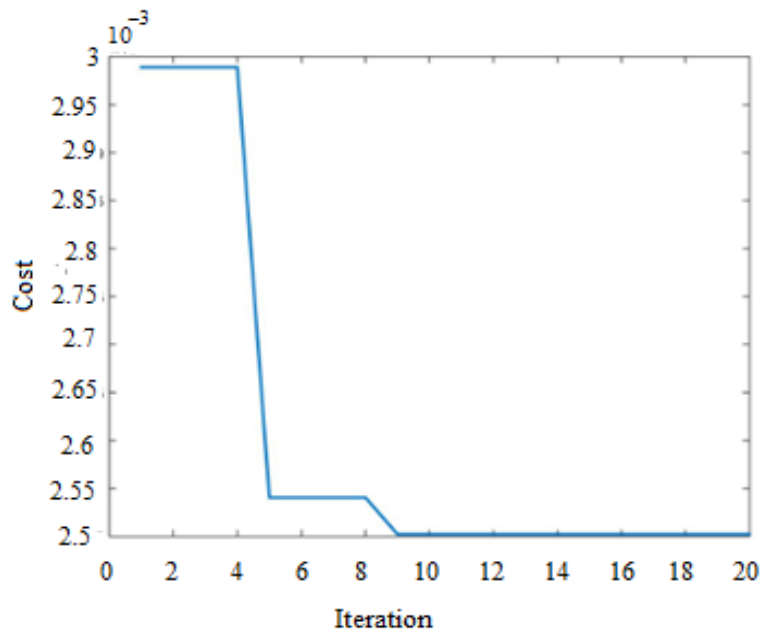

Figure 4. Best cost vs number of iterations

The network architecture in ACO-BP is shown in Figure 5. Here ten hidden layers are used and the optimized four parameters from MACO are used as input. In this optimization algorithm, the system considers all the parameters at the distributed side. It makes a decision based on the priority, cost and availability of the energy. When a high priority condition is met, the systems ignore all other parameters. The ANN helps us to schedule this non-continuous load and control the operation automatically for a long time. It also helps us to schedule the energy during peak demand. Comparison between different algorithms is shown in Table 2. From the table it is clear that the efficiency of the presented system is $99.63 \%$.

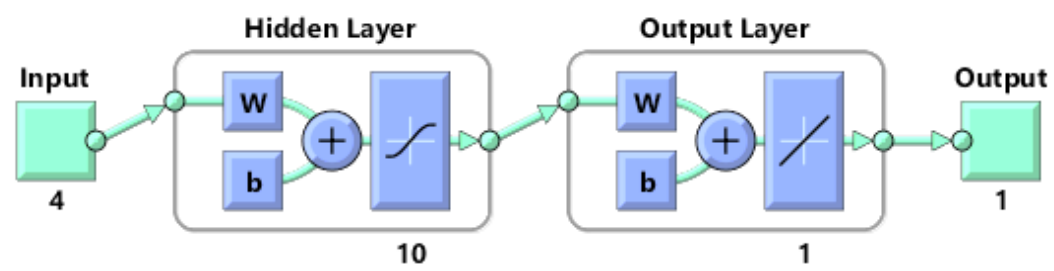

Figure 5. Network architecture of presented system 
Table 2. Comparison of various algorithms

\begin{tabular}{|c|c|c|c|c|c|c|c|}
\hline \multirow[b]{2}{*}{ Algorithms } & \multicolumn{3}{|c|}{ MSE } & \multicolumn{3}{|c|}{$\mathrm{R}$} & \multirow{2}{*}{$\begin{array}{c}\text { Accuracy } \\
\%\end{array}$} \\
\hline & Training & Validation & Testing & Training & Validation & Testing & \\
\hline $\mathrm{BP}$ & $5.95660 \mathrm{e}-3$ & $1.81126 \mathrm{e}-3$ & $1.61343 e-3$ & $9.94319 \mathrm{e}-1$ & $9.98478 \mathrm{e}-1$ & $9.97949 \mathrm{e}-1$ & 98.88 \\
\hline ACO-BP & $1.90762 \mathrm{e}-5$ & $2.49885 \mathrm{e}-2$ & $1.26032 \mathrm{e}-5$ & $9.99980 \mathrm{e}-1$ & $9.71110 \mathrm{e}-1$ & $9.99989 \mathrm{e}-1$ & 99.12 \\
\hline MACO-BP & $1.88456 \mathrm{e}-5$ & $2.51223 \mathrm{e}-2$ & $1.24022 \mathrm{e}-5$ & $9.99998 \mathrm{e}-1$ & $9.99998 \mathrm{e}-1$. & $9.72227 \mathrm{e}-1$ & 99.63 \\
\hline
\end{tabular}

\section{CONCLUSION}

Back Propagation algorithm has been used to adjust the connection weights in ANN. Basic BP algorithm is unstable and there is a possibility to get stuck in local optima. Here Levenberg-Marquardt algorithm is used in BP. In this work a global optimization algorithm(ACO) is hybridized with BP algorithm. There is a chance for convergence of initial connected weights selected for BP to a local optimum. The present work leads to get good initial connection weights through ACO and then pass the result to BP network for training. From the response of the system it is clear that the proposed hybrid training performance is better than the basic BP algorithm.

\section{REFERENCES}

[1] R. Hooshmand and M. Moazzami, "Optimal design of adaptive under frequency load shedding using artificial neural networks in isolated power system," Int. J. Electr. Power Energy Syst., vol. 42(1), pp. 220-228, Nov 2012.

[2] V. M. Joy and S. Krishnakumar, "Efficient Load Scheduling Method For Power Management," Int. J. Sci. Technol. Res., vol. 4, pp. 99-101, 2015.

[3] W. K. Foong, H. R. Maier, and A. R. Simpson, "Power plant maintenance scheduling using ant colony optimization: An improved formulation," Eng. Optim., vol. 40(4), pp. 309-329, 2008.

[4] D Harikrishna and N V Srikanth, "Dynamic Stability Enhancement of Power Systems Using Neural-Network Controlled Static-Compensator," TELKOMNIKA (Telecommunication Computing Electronic and Control), vol. 10(1), pp. 9 16, Mar 2012.

[5] Laxmi Devi Sahu and Satya Prakash Dubey, "ANN based Hybrid Active Power Filter for Harmonics Elimination with Distorted Mains," International Journal of Power Electronics and Drive System (IJPEDS), vol. 2(3), pp. 241 248, Sep 2012.

[6] V. Skorpil and J. Stastny, "Neural Networks and Back Propagation Algorithm," Electron. Bulg. Sozopol, pp. 173-176, Sep 2006.

[7] Q. Zeng and Z. Yang, "Integrating simulation and optimization to schedule loading operations in container terminals," Comput. Oper. Res., 36(6), pp. 1935-1944, Jun 2009.

[8] Sheela Tiwari1, et al, "Comparative Study Of Backpropagation Algorithms In Neural Network Based Identification Of Power System," Int. J. of Computer Science \& Information Technology (IJCSIT), vol. 5, pp. 93-107, Aug 2013.

[9] M. Suman, et al, "Solution of economic load dispatch problem in power system using lambda iteration and back propagation neural network methods," Int. J. Electr. Eng. Informatics, vol. 8, no. 2, pp. 347-355, Jun 2016.

[10] M. Marzband, et al, "Real time experimental implementation of optimum energy management system in standalone Microgrid by using multi-layer ant colony optimization," Int. J. Electr. Power Energy Syst., vol. 75, pp. 265-27, Feb 2016.

[11] M. A. Hossai et al., "Modified PSO algorithm for real-time energy management in grid-connected microgrids," Renew. Energy, vol. 136, pp. 746-757, 2019.

[12] E. Grossi and M. Buscema, "Introduction to artificial neural networks," European Journal of Gastroenterology and Hepatology, vol 12, no. 19, pp. 1046-1059, Dec 2007.

[13] M. S. B. Maind and M. P. Wankar, "Research Paper on Basic of Artificial Neural Network," Int. J. Recent Innov. Trends Comput. Commun., vol. 2(1), pp. 96-100, Jan 2014.

[14] Chen Lv, "Levenberg-Marquardt Backpropagation Training of Multilayer Neural Networks for State Estimation of a Safety-Critical Cyber-Physical System," IEEE Transactions on Industrial Informatics, vol 14(8), pp. 3436-3446, Aug 2018.

[15] H. W. Ressom, et al., "Ant colony optimization for biomarker identification from MALDI-TOF mass spectra," in Annual International Conference of the IEEE Engineering in Medicine and Biology - Proceedings, pp. 4560-4563, 2006.

[16] M. Serra and P. Venini, "On some applications of ant colony optimization metaheuristic to plane truss optimization," Struct. Multidiscip. Optim., vol. 32(6), pp. 499-506, Dec 2006.

[17] Peter Korošec et al, "The differential ant-stigmergy algorithm," Information Sciences, vol. 192, pp. 82-97, 2012.

[18] F. Heylighen, "Stigmergy as a universal coordination mechanism I: Definition and components," Cogn. Syst. Res., vol. 38, pp. 4-13, Dec 2015.

[19] Marco Dorigo and Thomas Stutzle, "Ant Colony Optimization: Overview and Recent Advances," Handbook of Metaheuristics (ISOR), vol. 272, pp. 311-351, Sep 2018.

[20] Aaron C. Zecchin, et al, "The Application of two ant colony optimisation algorithms to water distribution system optimisation," Mathematical and Computer modelling, vol. 44, pp. 451-468, Sep 2006. 
[21] I. Alaya, C. Solnon, and K. Ghédira, "Ant colony optimization for multi-objective optimization problems," in Proceedings - International Conference on Tools with Artificial Intelligence, vol. 1, pp. 450-457, Oct 2007.

[22] Abba Suganda Girsang, et al., "Fast Ant Colony Optimization for Clustering," Indonesian Journal of Electrical Engineering and Computer Science (IJEEI), vol. 12, pp. 78 86, Oct 2018.

[23] Li Yu, et al, "An Intelligent Scheduling Approach for Electric Power Generation," Chinese Journal of Electronics, vol. 27(6), pp. 1170 - 1175, Nov 2018.

[24] Sahib Khan and Tiziano Bianchi, "Ant Colony Optimization (ACO) based Data Hiding in Image Complex Region," International Journal of Electrical and Computer Engineering (IJECE), vol. 8(1), pp. 379-389, Feb 2018.

[25] C. Giri, S. Sarkar and S. Chattopadhyay, "A genetic algorithm based heuristic technique for power constrained test scheduling in core-based SOCs," 2007 IFIP International Conference on Very Large Scale Integration, Atlanta, GA, USA, pp. 320-323, 2007.

[26] Hao Luo, et al, "Hybrid flow shop scheduling considering machine electricity consumption cost," International Journal of Production Economics, vol. 146(2), pp. 423-439, Dec 2013.

\section{BIOGRAPHIES OF AUTHORS}
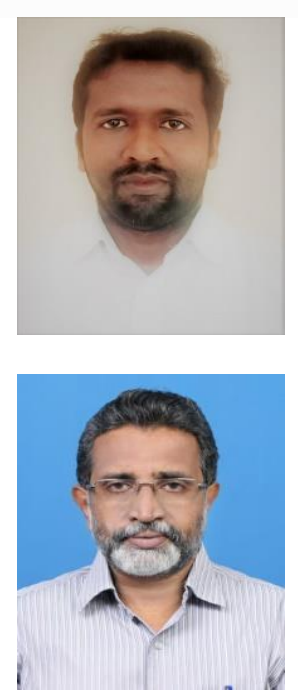

Vijo M. Joy completed his M.Sc. Electronics in 2006 from Mahatma Gandhi University, Kottayam. He got M.Tech. in Embedded System Design in 2011 from Indira Gandhi National Open University, Newdelhi. He has 10 years of teaching experience in Electronics subjects for graduates and post graduates. Currently he is working in the Department of Electronics, Aquinas College Edacochin, Cochin, Kerala, India. He is a research fellow in the Department of Electronics, M G University Research Center, Edapally, Cochin. His research interests are in the fields of Artificial Intelligents. He has published several research articles in reputed international journals.

Dr. S. Krishnakumar completed his M.Sc. in Physics with Electronics specialization in 1987 from Mahatma Gandhi University, Kottayam. He was awarded Ph.D. in Thin Film Devices in 1995 from Mahatma Gandhi University, Kottayam. He got M.Tech. in Computer Science from Allahabad Agricultural Institute - Deemed University (renamed as Sam Higginbottom Institute of Agriculture, Technology and Sciences) in 2006 and also obtained MCA from IGNOU in 2010. He qualified AICTE-UGC special examination for PG Engineering in 2018. He has 24 years of teaching experience in Electronics and Computer Science subjects for graduates and post graduates. He served as the Regional Director at the School of Technology and Applied Sciences (STAS), during the period 2013-17. Currently he is working in the STAS, Mahatma Gandhi University Research Centre, Edappally, Kochi-24, Kerala, India. His research interest fields include ANN, Analog circuit design and Image processing. Dr. S. Krishnakumar is an Associate member of Institute of Engineers, India. He was a member of Board of studies of University of Calicut and a member of Academic council of Mahatma Gandhi University, Kottayam. 